\title{
Comunicação
}

\section{A laparoscopia no diagnóstico das doenças intra-abdominais. Análise de 168 casos.}

\author{
N. A. Andreollo, J. de S. Coelho neto, L. R. Lopes, N. A. Brandalise, L. S. Leonardi \\ Disciplina de Moléstias do Aparel ho Digestivo do Departamento de Cirurgia e no Gastrocentro, Faculdade de Ciências Médicas da \\ Universidade Estadual de Campinas - UNICAMP, Campinas, SP.
}

\begin{abstract}
RESUMO - A laparoscopia tem sido mais freqüentemente indicada nos últi mos anos na complementação diagnóstica de afecções abdominais e na identificação de repercussões abdominais de doenças sistêmicas. Modernos equipamentos incluindo video-laparoscopia e o uso de agulhas de biópsias e outros instrumentais permitem elevada segurança e significante capacidade diagnóstica.

Овл етіvo. E mpregar a laparoscopia no diagnóstico de doenças abdominais e sistêmicas.

Métodos. Durante os últimos 9 anos, 168 doentes foram submetidos a laparoscopia e as principais indicações foram: ascites ( 43 casos - $25,5 \%$ ); doenças hepáticas ( 42 casos - $25 \%$ ); câncer gástri co (37 casos - 22\%); linfomas (17 casos - 10,1\%); tumores abdominais ( 9 casos - 5,4\%); tuberculose peritoneal ( 8 casos - 4,8\%); tumores hepáticos (6 casos - 3,6\%); cisto mesentérico ( 1 caso - 0,6\%) e outras doenças (5 casos - 3,0\%). Noventa e nove pacientes eram masculinos $(58,9 \%)$, com idade
\end{abstract}

\section{NTRODUÇÃO}

A primeiralaparoscopia diagnóstica foi realizada em 1902 simultaneamente em dois centros: em Dresden, por Kelling ${ }^{1}$ que visibilizou os órgãos intra-abdominais de um cão com auxílio de um cistoscópio e, em Petrogrado, por Ott ${ }^{2}$ que introduziu um espécul o no interi or do abdomem e levando a iluminação com auxílio de um espelho frontal visualizou os órgãos intracavitários.

Em 1923, J acobeus ${ }^{3}$ publicou sua experiência com aproximadamente 100 doentes, sendo que o primeiro deles era portador de ascite e foi examinado em 1910. A seguir, outros autores contribuíram para o desenvolvimento da laparoscopia. Assim, Fervers ${ }^{4}$, em 1933, introduziu o uso do gás carbônico como agente insuflante seguro para a cavidade abdominal. Veress ${ }^{5}$, em 1938, desenvolveu a agulha com obturador rombo, a qual é utilizada até hoje para criar o pneumoperitoneo e inclusive leva o seu nome, tornando o procedimento muito seguro. Kalk hepatologista de renome, em 1951, introduz o uso de trocartes e outros instrumentos úteis na Iaparoscopia6.

Nas décadas de 70 e 80 surgiram técnicas variando de 9 a 78 anos (47,6 anos). Biópsias hepáticas foram realizadas em 92 casos $(54,7 \%)$ e outras biópsias tumorais em 26 casos (15,4\%).

Resultados. O diagnóstico da doença foi estabelecido ou confirmado em 145 casos (86,3\%). E m 25 casos de câncer gástrico $(67,5 \%)$, a laparoscopia contra-indicou a laparotomia em função de detecção de doença avançada. Dois pacientes apresentaram hemorragia $(1,2 \%)$ após biópsias hepáticas e a laparotomia foi imediatamente indicada. Um doente, cujo diagnóstico era de lupus sistêmico, apresentou abscesso abdominal, broncopneumonia e faleceu $(0,6 \%)$.

ConCLUSÃo. O procedimento tem pequeno número de complicações e quando empregado como método diagnóstico complementar, evita a laparotomia e agiliza medidas terapêuticas.

UNITERMOS: Laparoscopia. Doenças intra-abdominais. Estadiamento.

radiológicas não-invasivas de imagem (ultrassonografia, tomografia etc.) e o método não foi muito difundido entre os gastrenterologistas e cirurgiões gerais, sendo mais utilizado pelos ginecologistas. Llanio et $\mathrm{al}^{7}$ foram pioneiros em divulgar o método como eficaz em avaliar urgências abdominais. Foi pouco valorizado durante alguns anos e na década de 90 novamente passou a ser muito empregado, desta vez no diagnóstico do abdômem agudo, traumatismos e ferimentos abdominais ${ }^{8}$. A partir de 1986, após Cuschieri ter comunicado a realização de colecistectomia através de vídeo-laparoscopia, a laparoscopia deixou de ser simplesmente diagnóstica para ser intervencional nas doenças intra-abdominais e do aparel ho digestivo $0^{6,9-11}$.

Assim, aproximadamente um século após ter sido introduzida como recurso diagnóstico, o procedi mento volta a ser muito utilizado e valorizado, agora com modernos e mais avançados instrumentos e equipamentos, contribuindo para o tratamento de várias doenças. O objetivo do presente trabal ho é mostrar a experiência do emprego deste método no Hospital das Clínicas da UNICAMP. 


\section{CASUÍSTICA E METOdOLOGIA}

Durante os últimos nove anos, 168 doentes foram submetidos à laparoscopia na Disciplina de Moléstias do Aparelho Digestivo, Departamento de Cirurgia e Gastrocentro da FCM/UNICAMP, para diagnóstico de doenças intra-abdominais. E ram 99 masculinos (58,9\%) e 69 femininos (41,1\%) com idade variável de 9 a 78 anos (média 47,6 anos).

A laparoscopia foi indicada após terem sido realizados os princi pais exames relativos à doença em que estava sendo diagnosticada, tais como ultrassonografia, punção de ascite, tomografia e outros exames complementares. As indicações da laparoscopia estão discriminadas na tabela 1.

O tempo, a atividade de protrombina e a contagem de plaquetas no sangue periférico foram os exames laboratoriais sistematicamente solicitados. O procedimento foi realizado nos doentes com no mínimo $50 \%$ de atividade de protrombina e 80.000 plaquetas. E m 30 doentes (17,8\%) a laparoscopia foi realizada mediante uso de anestésico local (xylocaína a 2\%) para introdução do trocarte na parede abdominal, acompanhada de sedação endovenosa do doente. Todos os demais foram realizados com anestesia geral.

A técnica de laparoscopia empregada foi a convencional ${ }^{9}$. Incisão transversal supraumbelical de cerca de $1,5 \mathrm{~cm}$ e introdução de agulha de Veress para realização de pneumoperitoneo com CO2. Para realização do pneumoperitoneo, foi utilizado insuflador a pressão da marca Wisap. Após distensão abdominal satisfatória, retirada da agulha de Veress e introdução com cui dado de trocarte de $10 \mathrm{~mm}$ de diâmetro e a seguir col ocação da ótica de 0․ Para iluminação foi empregada fonte de luz Olympus CLE-F 10. Dispondo-se de equipamentos de video-laparoscopia, a conexão da microcâmera à ótica, permitirá visualização através de um monitor, ampliando e mostrando imagens mais detalhadas.

O procedimento inicia-se com o exame minucioso de todo o peritoneo parietal, de ambos os lobos hepáticos, do baço, grande epíplon e da superfície das alças intestinais e demais órgãos.

As biópsi as hepáticas for am realizadas com aguIha de Silverman e outros instrumentos tais como afastador de fígado, agulha para biópsia de peritoneo e outras pinças auxiliares, que foram introduzidas na cavidade abdominal quando necessárias. O local preferido para biópsia hepática foi o lobo esquerdo. Os doentes portadores de ascite vol umosas foram drenados durante o procedimento, utilizando-se aspirador apropriado, para adequada visualização de toda a cavidade peritoneal.

A video-laparoscopia foi realizada em 25 casos

\begin{tabular}{|c|c|c|}
\hline Diagnóstico & № casos & $\%$ \\
\hline $\begin{array}{l}\text { Ascite a esclarecer (exsudatos/transudatos/ } \\
\text { quilosa/hemorrágica }\end{array}$ & 43 & 25,5 \\
\hline $\begin{array}{l}\text { Hepatopatias (hepatites/cirroses } \\
\text { Budd-Chiari, etc.) }\end{array}$ & 42 & 25 \\
\hline $\begin{array}{l}\text { Câncer gástrico (Carcinomatose/metástase } \\
\text { hepáticas/linfonodomegalia) }\end{array}$ & 37 & 22 \\
\hline Linfomas (estadiamento/biópsias) & 17 & 10,1 \\
\hline $\begin{array}{l}\text { Tumores abdominais (mesentéricos/ } \\
\text { retroperitoneais/outros) }\end{array}$ & 9 & 5,4 \\
\hline Tuberculose peritoneal & 8 & 4,8 \\
\hline $\begin{array}{l}\text { Tumores hepáticos (tumores primários/ } \\
\text { metástases) }\end{array}$ & 6 & 3,6 \\
\hline Cisto mesentérico & 1 & 0,6 \\
\hline $\begin{array}{l}\text { Outras doenças (Lupus/Aids/Câncer de } \\
\text { pâncreas/Doença inflamatória intestinal) }\end{array}$ & 5 & 3,0 \\
\hline Total & 168 & 100 \\
\hline
\end{tabular}

desta casuística, utilizando-se equipamento convencional para cirurgia vídeo-laparoscópica ${ }^{11}$.

\section{RESULTADOS}

O diagnóstico da doença intra-abdominal foi estabelecido ou confirmado pela laparoscopia em 145 pacientes $(86,3 \%)$. Nos portadores de câncer gástrico a laparoscopia demonstrou que 25 doentes $(67,5 \%)$ eram portadores de carcinomatose peritoneal ou tinham tumores irressecáveis, contraindicando portanto a laparotomia exploradora.

As biópsias hepáticas foram realizadas em 92 casos $(54,7 \%)$ e biópsias de outros tumores em 26 casos $(15,4 \%)$. Dois doentes que tinham atividade de protrombina aproximadamente $60 \%$ apresentaram hemorragia após a realização de biópsias hepáticas e a laparotomia foi imediatamente indicada (1,2\%). Um destes pacientes apresentou evolução satisfatória após a hemostasia do local de sangramento e o outro, o qual era portador de lupus sistêmico grave, evoluiu com abscessos intra-abdominais, broncopneumonia e sepses, falecendo $(0,6 \%)$ cerca de 10 dias após a laparoscopia.

Como complicação tardia, um dos doentes que apresentava ascite evoluiu com perda continuada e crônica deste líquido pel o local de introdução do trocarte, sendo necessário uma exploração cirúrgica para ressutura da parede abdominal.

\section{DISCUSSÃO}

A laparoscopia é um procedimento invasivo de diagnóstico, bem tolerado e seguro em mãos 
experientes. As principais indicações eletivas do método são em oncologia, nas biópsias hepáticas, nas ascites, em febre de origem indeterminadas e outras $^{12}$. Em oncologia as indicações são no estadiamento, na avaliação da extensão, operabilidade e ressecabilidade dos tumores, na avaliação de metástases e na realização de biópsias com aguIhas ou pinças apropriadas ${ }^{11,13}$. A biópsia hepática, através da laparoscopia é dirigida e muito mais segura que a biópsia percutânea, visto que é realizada sob visão direta e com controle da intensidade da hemorragia.

Em portadores de ascite cuja etiologia não foi estabelecida com os exames habituais, a laparoscopia está indicada. Cuidados especiais devem ser tomados nestes doentes, tais como: reposição vol êmica após aspiração do líquido ascítico, introdução cuidadosa da agulha de Veress e trocartes devido risco de lesão e hemorragia de veias colaterais, pneumoperitoneo lento para evitar a formação de bolhas gasosas e fechamento cuidadoso dos orifícios na parede abdominal para que não ocorra fístula e vazamento de líquido ascítico ${ }^{14}$.

$\mathrm{N}$ os doentes com febre de origem indeterminada em que não foi possível chegar ao diagnóstico final, a laparoscopia está indicada com o objetivo de diagnosticar linfomas ou outras doenças ${ }^{6,13}$.

Em situações de emergência, a laparoscopia poderá ser empregada nos ferimentos penetrantes abdominais, nos traumas abdominais fechados, na suspeita de isquemia intestinal e no abdomem agudo inflamatório sem causa definida, sempre no sentido de evitar laparotomias desnecessárias 7,8 .

A laparoscopia sendo método invasivo, está sujeita à complicações que o cirurgião ou o gastrenterol ogista devem conhecer e saber tratá-las. São incl uídas nas compli cações comuns a todos os procedimentos cirúrgi cos, tais como: anestésicas, sangramento e infecção pós-operatória. Além disso, existem as complicações específicas do método, decorrentes do pneumoperitoneo, da agulha de Veress e trocartes e de outros procedimentos diagnósti $\cos ^{9,15}$.

As complicações anestési cas são raras, porém o anestesista deve estar sempre atento, pois o aumento da pressão intra-abdominal secundário ao pneumoperitoneo, a posição de Trendelenburg, cardiopatias e pneumopatias pré-existentes, contribuem para favorecer o surgimento de arritmias cardíacas, hipoventilação, hipóxia, acidose e outros efeitos colaterais. O procedimento pode ser também realizado com anestesia local e sedativos endovenosos, preferencialmente na presença de um anestesista. Assim mesmo, a cabeça do doente deve ser mantida mais elevada para prevenir aspirações pulmonares secundárias às náuseas e vômitos que são freqüentes, quando o exame é feito com este tipo de anestesia ${ }^{6,15}$.

As complicações significantes do pneumoperitoneo também são raras, ocorrendo em menos que $1 \%$ dos casos. É importante saber que a insuflação intra-abdominal de gás causa certas mudanças fisiológicas na hemodinâmica e função pulmonar de todos os pacientes. O aumento da pressão intra-abdominal resulta em aumento da pressão venosa central dos batimentos e do débito cardíaco e da pressão arterial média. O gás carbônico (CO2), o gás mais freqüentemente utilizado, pode causar aumento do pCO2 e diminuição do pH arterial, resultando em acidose metabólica e respiratória. A embolização pelo $\mathrm{CO} 2$, a complicação mais grave e fatal, felizmente é muito rara. $O$ pneumotórax e o enfizema subcutâneo são outras complicações que podem ocorrer ${ }^{15}$.

As complicações decorrentes da inserção dos trocartes são a hemorragia e perfuração de vísceras. A hemorragia, decorrente de lesão de veias da parede abdominal, especialmente as periumbelicais que estão dilatadas e com abundante circulação colateral secundária à hi pertensão portal em portadores de cirrose hepática e outras hepatopatias. Na maioria das publicações sua incidência é estimada em $0,2 \%$. As perfurações intestinais envolvem principalmente o cólon transverso e menos comumente o intestino del gado. Ocorrem em incidência maior em doentes com cirurgia abdominal anterior e doentes com aderências intestinais secundárias à peritonite e tuberculose intestinal. É importante diagnosticar a perfuração etratá-la adequadamente em tempo, evitando graves complicações ${ }^{14,15}$.

Outras complicações durante a laparoscopia incluem perfurações intestinais e traumatismos de outras vísceras como fígado, baço, bexiga, mesentério, etc, causadas principalmente pela própria ótica, outras pinças ou instrumentos introduzidos na cavidade peritoneal ${ }^{14,15}$.

As complicações mais freqüentes decorrentes da biópsia hepática são a hemorragia e a peritonite biliar ${ }^{14,15}$. Brhul ${ }^{16}$ em 1966 revisou 63.845 laparoscopias e relata ocorrência de peritonite biliar em 0,05\%, resultando em 0,013\% de mortalidade. Entretanto, a hemorragia, registrada na frequência entre 0,02 a $0,2 \%$, na maioria das séries publicadas. A hemorragia raramente ocorre se for respeitado o limite de $50 \%$ de atividade de protrombina e contagem de plaquetas sanguín eas $>80.000 / \mathrm{mm}^{3}$. O l obo esquerdo é mais apropriado para bi opsiar, pois no caso de hemorragia, é facilmente atingido e passível de 
compressão externa na parede abdominal $\left.\right|^{9,15}$.

As complicações tardias pós-laparoscopias também são descritas e incluem implantes metastáticos nos locais de inserção de trocartes e aguIhas de biópsias, hérnias nos locais de incisão e infecção intra-abdominal. A pesar disso, é um método seguro, com incidência gl obal muito baixa de complicações. Revisões extensas incluindo mais de 200.000 laparoscopias registram 0,05\% de morbidade e mortalidade, não elevando o tempo de hospitalização ${ }^{15}$.

Marti-Vicenti et al. ${ }^{17}$ em recente revisão de 8.915 Iaparoscopias referem $0,60 \%$ de acidentes, sendo metade delas decorrentes de instrumentação e biópsias hepáticas. A mortalidade global foi de $0,11 \%$ como sequela de hemorragia incontrolável, sendo a metade delas secundárias à biópsias hepáticas. Entretanto, Kane \& $\mathrm{Krej}^{18}$ revisaram 603 laparoscopias de 17 centros americanos e referem mortalidade de $0,49 \%$.

O valor, a eficácia e a segurança da laparoscopia é notório quando se está diante de doente que não apresenta condições clínicas satisfatórias para ser submetido à laparotomia exploradora. Além disso, estudos prospectivos já demonstraram que em muitos casos a tomografia computadorizada, a ultrassonografia e a cintilografia são exames limitados, não contribuindo para o diagnóstico definitivo ${ }^{10,11}$.

A laparoscopia, segundo $\mathrm{N}$ ord \& $\mathrm{B}_{\text {oyd }}{ }^{10}$ e Puccio et al. ${ }^{19}$, pode contribuir para o diagnóstico definitivo em até $90 \%$ dos casos, sendo que os demais $10 \%$ mostram resultados normais ou inespecíficos. $\mathrm{Na}$ presente casuística o diagnóstico foi estabelecido em $86,3 \%$.

Nas doenças hepáti cas o diagnóstico é estabeleci do em prati camente $100 \%$ dos casos, pois permite a realização de biópsias com segurança ${ }^{14}$. Nos tumores hepáticos, $\mathrm{N}$ ord $\&$ B oyd ${ }^{11}$ relatam experiência de TJ Babineau et al. (1993), em que a laparoscopia revel ou irressecabilidade dos tumores em 45\% dos casos, evitando laparotomias desnecessárias. Lightdal $\mathrm{e}^{13}$ demonstra a eficácia da laparoscopia no estadiamento de tumores gastrintestinais, como carcinoma pancreático, carcinoma gástrico, linfomas e outros em comparação com os métodos radiológicos de imagem.

$\mathrm{Na}$ casuística apresentada, em $56 \%$ dos casos de carcinoma gástrico, a laparoscopia realizada pré-operatoriamente demonstrou inoperabilidade, evitando laparotomias desnecessárias. D'ugo et $a l .{ }^{20}$ enfatizam que é um procedimento fundamental com eficácia de $68,6 \%$ na avaliação da ressecabilidade destas neoplasias. Molloy et $a^{21}$ mostram resultados semel hantes.
O único óbito registrado na série apresentada $(0,6 \%)$ decorreu do fato de que a hemorragia, após a biópsia hepática, em doente grave e portador de lupus, mesmo tendo sido prontamente atendido, apresentou outras complicações, vindo a falecer em curto período de tempo.

A el evada acuidade e eficácia da laparoscopia a tornam atrativa, no sentido de que sua utilização contribui para diminuir os gastos e custos hospitalares, não apenas na economia de exames radiológicos, mas encurtando o tempo de hospital ização do doente. O procedimento pode ser realizado com anestesia local eatémesmo em sal a de endoscopia ${ }^{10,19}$.

A laparoscopia, nestes anos todos de evolução, sem dúvida trouxe subsídi os importantes e fundamentais para o desenvolvimento da cirurgia por video-laparoscopia, cada vez mais difundi da a partir de 1990. Por outro lado, aproveitando hoje a experiência e os equipamentos da cirurgia por video-laparoscopia, o emprego da video-laparoscopia diagnóstica tem contribuído ainda mais para ampliar os horizontes do método.

A video-laparoscopia diagnóstica permite uma visão ampliada da cavidade abdominal e, além disso, auxiliada por outros instrumentos e pinças introduzidas através de trocartes, permite a realização de biópsias, exérese de linfonodos, ressecções de pequenos fragmentos de tecidos para exames e cauterizações, aumentando a eficácia do exame e di minuindo a incidência de compli cações ${ }^{9}$.

Assim, pode-se concluir, que a laparoscopia tem seu lugar bem definido no arsenal médico. É um método moderno, de baixo custo, seguro e eficaz, de fácil execução, contribuindo para o diagnóstico das doenças intra-abdominais e agilizando medidas terapêuticas.

\section{SUMMARY}

\section{The laparoscopy in the diagnoses of intra-} abdominal diseases. Analysys of 168 cases.

The laparoscopy has been more and more fre quently indicated in the last years, for the complementary diagnosis of intraabdominal diseases and abdominal repercussion of systemic diseases. Modern equipaments including videolaparoscopy and the use of either forceps or biopsy needle permit higher safety and significant diagnostic capacity.

PURPOSE. To perform laparoscopy for the diagnosis of intraabdominal and sistemic diseases.

Methods. During the last 9 years 168 patients were submitted to laparoscopy and the main indications were: ascites - 43 cases (25,5\%); liver 
diseases - 42 cases ( $25 \%$ ); gastric cancer - 37 cases (22\%); Iymphoma - 17 cases (10,1\%); abdominal tumour - 9 cases (5,4\%); peritoneal tuberculosis - 8 cases $(4,8 \%)$; liver tumour - 6 cases $(3,6 \%)$; mesenter i c cyst - 1 case $(0,6 \%)$ and other di seases - 5 cases $(3,0 \%)$. Ninety nine patients were male $(58,9 \%)$ aging from 9 to 78 years old (median 47,6 years). Liver bi opsies were performed in 92 cases $(54,7 \%)$ and other tumour biopsies in 26 cases $(15,4 \%)$.

RESULTS. The diagnosis of the diseases were established or confirmed by laparoscopy in 145 patients $(86,3 \%)$. In 25 cases of gastric cancer $(67,5 \%)$ laparoscopy contraindicated the laparotomy, owing to advanced disease. Two patients presented bleeding $(1,2 \%)$ after liver biopsies and laparotomy was immediately indicated. One of them, whose diagnosis was systemic lupus, pre sented abdominal abscess, bronchopneumony and died $(0,6 \%)$.

ConcLusion. Laparoscopy has small number of complications, and when employed as a diagnostic complementary method therapeutic procedures, avoids laparotomies and accelerate therapeutic procedures. [Rev Ass Med Brasil 1999; 45(1): 34-8.]

KEY WORDS: Laparoscopy. I ntraabdominal diseases. Staging.

\section{REFERÊNCIAS BIBLIOGRÁFICAS}

1. Kelling G. Zur Coelioskopie. Arch Klin Chir 1923; 126:226-29.

2. Ott O. Die Direkte Beleuchtung der Bauchhohle, der Harnblase, des Dickdarms und des Utgerus zu Diagnostichen Zwecken. Rev Med Tcheque (Prague) 1909; 2:27.

3. J acobeus HC. Kurze U bersicht uber meine Erfahrungen Mit der Laparoskopie. Munch Med Wschr 1923; 58:2017-19.

4. Fervers J. Die Laparoskopie mit dem Zystoskope mit dem Zystoskope. MedscheKIin 1933; 29:1042-45.

5. Veress J. Neues Instrument zue Ausfuhrung von Brust Oder Bauchpunktionen. Dtsch Med Wschr 19312538;41:1480-81.
6. Sackier J M \& Berci G. Diagnostic and Interventional Laparoscopy for the General Surgeon. Contemporary Surgery 1990; 37:15-26.

7. Llanio R, Sotto A, J imenez G, Quintero M, Ferret O, Manso E, Nodarse E. Resultados obtenidos con la laparoscopia de urgencia. Reporte de 1509 casos. Rev Cub Circ 1973; 12:23-27.

8. Zantut LF, I vatury RR, Smith RS, Kawahara NT, Porter J M, Fry WR, Poggetti R, Birolini, D, Organ CH J r. Diagnosticand Therapeutic Laparoscopy for Penetrating Abdominal Trauma: a Multicenter Experience. J Trauma 1997;42:825-829.

9. Soper NJ , Odem RR, Clayman RV, M cdougall EM. Essentials of Laparoscopy. Quality Medical Publishing, la ed. Saint Louis, USA, 1994. pp 1-285.

10. Nord HJ \& Boyd WP. Diagnostic Laparoscopy. Endoscopy 1994; 26:126-133.

11. Bogen GL, Mancino AT, Scott-Conner CEH. Laparoscopy for Staging and Palliation of Gastrointestinal Malignancy. Surg Clin N Am 1996; 76:557-569.

12. Henning $\mathrm{H}$. Indications and Contraindications of Diagnostic Laparoscopy. Endoscopy 1992; 674-75.

13. Lightdale CJ . Laparoscopy for Cancer Staging. Endoscopy 1992; 24:682-86.

14. Boyce HW. Diagnostic Laparoscopy in Liver and Biliary Disease. Endoscopy 1992; 24:676-81.

15. Nord HJ . Complications of Laparoscopy. Endoscopy 1992; 24:693-700.

16. Bruhl W. Zwischenfalle und Komplikationen bei der Laparoskopie und Gezielten Leberpunktion. Dtsch Med Wschr 1966; 91:2297-99.

17. Marti-Vicente A, Garcia V, Toro H, Seres I, Enriquez J , Vilardell F. Acidentes Y Complicaciones de la Laparoscopia. Revision de 8.915 casos. Rev Esp Enf Digest 1992;82:411-17.

18. Kane MG \& Krejs GJ . Complications of Diagnostic Laparoscopy in Dallas: a 7-year prospective study. Gastrointest Endosc 1984; 30:237.

19. Puccio J E, Kulesza MD, Gordon SC. Should Gastroenterologists Perform Diagnostic Laparoscopy in the Operating Room? AmJ Gastroenterol 1992;87:A1303.

20. D'U go DM, Coppola R, Persiani R, Ronconi P, Caracciolo F, Picciocchi A. I mmediately Preoperative Laparoscopic Staging for Gastric. Cancer. Surg Endosc 1996;10:996-999.

21. Molloy RG, McCarteny J S, Anderson J R. Laparoscopy in the Management of Patients with Cancer of the Gastric Cardia and Esophagus. Br J Surg 1995; 82: 352-4. 